\title{
Multi-scale energy-based failure modeling of bond pad structures
}

\author{
O. van der Sluis ${ }^{1,2 *}$, R.B.R. van Silfhout ${ }^{1}$, R.A.B. Engelen ${ }^{1}$, W.D. van Driel ${ }^{2,3}$, and G.Q. Zhang ${ }^{2,4}$ \\ ${ }^{1}$ Philips Applied Technologies, High Tech Campus 7, 5656 AE Eindhoven, The Netherlands \\ ${ }^{2}$ Department of Precision and Microsystem Engineering, Delft University of Technology, the Netherlands \\ ${ }^{3}$ NXP Semiconductors, IMO-BE Innovation BY1.055 Gerstweg 2, 6534 AE Nijmegen, The Netherlands \\ ${ }^{4}$ NXP Semiconductors, High Tech Campus 60, 5656 AE Nijmegen, The Netherlands
}

\begin{abstract}
Thermo-mechanical reliability issues have been identified as major bottlenecks in the development of future microelectronic components. This is caused by the following technology and business trends: (1) increasing miniaturisation, (2) introduction of new materials, (3) shorter time-to-market, (4) increasing design complexity and decreasing design margins, (5) shortened development and qualification times, (5) gap between technology and fundamental knowledge development [22]. It is now well established that for future CMOS-technologies (CMOS065 and beyond), low- $k$ dielectric materials will be integrated in the back-end structures [8]. However, bad mechanical integrity as well as weak interfacial adhesion result in major thermo-mechanical reliability issues. Especially the forces resulting from packaging related processes such as dicing, wire bonding, bumping and molding are critical and can easily induce cracking, delamination and chipping of the IC back end structure when no appropriate development is performed [4]. The scope of this paper is on the development of numerical models that are able to predict the failure sensitivity of complex three-dimensional multi-layered structures while taking into account the details at the local scale of the microelectronic components by means of a multi-scale method. The damage sensitivity is calculated by means of an enhanced version of the previously introduced Area Release Energy (ARE) criterion. This enhancement results in an efficient and accurate prediction of the energy release rate (ERR) at a selected bimaterial interface in any location. Moreover, due to the two-scale approach, local details of the structure are readily taken into account. In order to evaluate the efficiency and accuracy of the proposed method, several two-dimensional and three-dimensional benchmarks will be simulated. The paper focusses on the enhanced ARE method, including several two- and three-dimensional benchmarks.
\end{abstract}

\section{Introduction}

The introduction of new low $-k$ materials, such as Black Diamond-I (CMOS090) and Black Diamond-I(x) (for CMOS065), results in major reliability issues. Especially the latter material, being porous, will drastically reduce the thermo-mechanical performance of the IC stack [4]. Indeed, Liu et al. [14] prove in their numerical analyses that the crack driving force increases with decreasing stiffness of the low- $k$ material. Finite element modeling combined with experimental observations and validations, pro-

\footnotetext{
*corresponding author: olaf.van.der.sluis@philips.com
}

vides a way to gain more fundamental knowledge and ultimately, to understand, predict and prevent reliability issues. However, numerical methods and models that are readily available in commercial finite element packages, are currently not sufficient to model the mentioned phenomena accurately and efficiently. First, due to the inherent scale-difference between application ( $[\mathrm{cm}]$ to $[\mathrm{mm}]$ ) and smallest geometry detail ([ $\mu \mathrm{m}]$ to $[\mathrm{nm}])$, a multi-scale method should be used to cover these length-scale differences in an appropriate way. Second, delamination of three-dimensional multi-layered structures should be taken into account. Third, the three-dimensional geometry of the back-end is complex and the individual material and interface properties should be measured accurately.

For this purpose, we have developed a numerical framework that takes into account (i) the scale difference by means of a homogenization step, (ii) delamination sensitivity between the different materials, and (iii) the complex three-dimensional geometry of the bond pads [6, 18]. Especially the introduction of an energy-based failure criterion, the Area Release Energy (ARE) method has proven to be a rather efficient way to evaluate the damage sensitivity of complex multi-material structures in three dimensions. It has also been proven that interface stresses do not provide meaningfull results with respect to the delamination sensitivity thereby confirming the applicability of energy-based criteria, based on principles of fracture mechanics. However, the original ARE method does not calculate the value for the energy release rate, even though it is an energy-based value. The calculated values can therefore not be compared with measured interface strengths. To this end, numerical fracture mechanics methods, like the $J$-integral method [11] or the virtual crack closure technique (VCCT) [17] can be employed. However, these methods only provide accurate values for the energy release rate when using proper crack tip meshes, proposed by Barsoum [3] for the $J$-integral method and by Smith and Raju [19] for the VCCT method. As a result, generation of three-dimensional models including these typical crack tip meshes is a cumbersome task to evaluate even one single crack with given location, size and geometry in a structure, as is performed by Wang et al. [21] and Lui et al. [14]. These shortcomings have motivated the development of the enhanced ARE method. This method, based on a two-scale scheme, facilitates accurate calculation of ERR values at an arbitrary location while preserving the main advantage of the original ARE method: efficient damage sensitivity analysis of complete interfaces within gen- 
eral three-dimensional structures. The method will be explained in more detail in the next section, after which several analytical and numerical benchmarks will be solved in order to assess the proposed method.

\section{Interface fracture mechanics}

In order to give a more fundamental basis to the concepts that will be discussed in this paper, a short outline of interfacial fracture mechanics will be given. For more detail, the reader is referred to $[10,15]$.

Although the strength of the elastic singular stress field near the tip of an interface crack has the usual $\sqrt{r}$ singularity, it also exhibits an oscillatory behavior near the crack tip region. This behavior is a characteristic feature of interface cracks and results in a coupling between the stress intensity factors $K_{1}$ and $K_{2}$ :

$$
\sigma_{i j}=\frac{\Re\left(\mathbf{K} r^{i \varepsilon}\right)}{\sqrt{2 \pi r}} \tilde{\sigma}_{i j}^{\mathrm{I}}(\theta, \varepsilon)+\frac{\Im\left(\mathbf{K} r^{i \varepsilon}\right)}{\sqrt{2 \pi r}} \tilde{\sigma}_{i j}^{\mathrm{II}}(\theta, \varepsilon)
$$

where the complex stress intensity factor $\mathbf{K}=K_{1}+i K_{2}$, which uniquely characterizes the singular stress field [15]. The local polar coordinates for a coordinate system located on the crack front in planes perpendicular to the crack front are represented by $r$ and $\theta$. The functions $\tilde{\sigma}_{i j}^{\mathrm{I}}(\theta, \varepsilon)$ and $\tilde{\sigma}_{i j}^{\mathrm{II}}(\theta, \varepsilon)$ describe the angular distribution of the stresses around the crack tip and are given in Rice and Shih [16]. The bimaterial index $\varepsilon$ is defined by

$$
\varepsilon=\frac{1}{2 \pi} \ln \left(\frac{1-\beta}{1+\beta}\right)
$$

where $\beta$ is one of the Dundur's elastic mismatch parameters and is related to the shear moduli $\mu_{i}$

$$
\beta=\frac{\mu_{1}\left(\kappa_{2}-1\right)-\mu_{2}\left(\kappa_{1}-1\right)}{\mu_{1}\left(\kappa_{2}+1\right)+\mu_{2}\left(\kappa_{1}+1\right)}
$$

where $\kappa_{i}=3-4 \nu_{i}$ for plane strain problems. The subscripts 1 and 2 are associated with the different materials on either side of the interface. The mode mixity is defined as

$$
\tan \psi=\frac{\Im\left(\mathbf{K} \ell^{i \varepsilon}\right)}{\Re\left(\mathbf{K} \ell^{i \varepsilon}\right)}
$$

where $\ell$ is a reference length which has to be provided for each calculated or measured $\psi$-value. The energy release rate (ERR) for an interface is given by

$$
G=\frac{1-\beta^{2}}{E_{*}}\left(K_{1}^{2}+K_{2}^{2}\right) \text { with } \quad \frac{1}{E_{*}}=\frac{1}{2}\left(\frac{1}{\bar{E}_{1}}+\frac{1}{\bar{E}_{2}}\right)
$$

where $\bar{E}_{i}=E_{i} /\left(1-\nu_{i}^{2}\right)$. The crack tip opening displacements (CTOD) are asymptotically speficied by $\mathbf{K}$ according to

$$
\delta_{2}+i \delta_{1}=\frac{8}{(1+2 i \varepsilon) \cosh \pi \varepsilon} \frac{\mathbf{K} r^{i \varepsilon}}{E_{*}} \sqrt{\frac{r}{2 \pi}}
$$

In our finite element analyses, the ERR has been chosen as crack driving force parameter, which will be calculated by the $J$-integral method [11]. For accurate calculation of the $J$-integral value, the singularity, denoted by $r^{\lambda}$, where $r$ is the distance from the crack tip and $\lambda$ is the order of the singularity, should be captured properly. For homogeneous materials, for which $\lambda=0.5$, Barsoum [3] has shown that the singularity can be described exactly when using so-called quarter point elements. However, as pointed out by Abdel-Wahab and de Roeck [1], these elements cannot be used for $\lambda$-values other than 0.5 , unless a fine mesh is used. This is confirmed by He et al. [9], who show that for interface cracks, convergence upon mesh refinement is obtained, however, with extremely fine meshes for high elastic mismatch values.

\section{Enhanced ARE approach}

As was already mentioned in the Introduction, one of the major disadvantages of fracture mechanics analysis is the necessary assumption of the location, shape and size of an initial defect [5]. Especially in the numerical analysis of complex three-dimensional geometries, inserting these defects into the model is quite a cumbersome task. The reason for this is the crack tip mesh requirement, which, particularly in three dimensions, is not straightforward to insert in these models. Consequently, most of the analyses are restriced to one or several crack location instead of complete interfaces $[14,21]$. This has motivated the development of the so-called Area Release Energy (ARE) method $[6,18]$. The ARE value predicts the delamination sensitivity of interfaces without knowing a priori the exact location of the delamination. Instead, the amount of energy is calculated that is released upon delamination for any position along any interface. As a result, an instant overview of the critical areas within any interface through a contour map is given thereby providing a direct comparison between different bond pad designs. The ARE value is calculated for each node $i$ in an interface according to

$$
G_{i}^{\mathrm{ARE}}=\frac{1}{2 A_{i}} \sum_{j=1}^{n} \mathbf{F}_{j}^{T}\left[\mathbf{u}_{j}\right]
$$

in which $n$ is the number of nodes that are released within the area $A_{i}$ around node $i$ (in 2D: $A_{i}=2 \ell t$; in 3D: $A_{i}=\pi \ell^{2}$, where $2 \ell$ is the size of the defect), $\mathbf{F}_{i}$ is the force vector acting on the nodes before release, $\left[\mathbf{u}_{i}\right]$ is the crack opening displacement vector and $t$ the thickness. For more information about the ARE method, the reader is referred to $[6,18]$. Although this method provides a flexible way to capture the failure sensitivity of complex threedimensional multi-material structures in the sense that critical locations are automatically identified without assuming a predefined location, several limitations have been identified: (i) due to the fact that the energy is calculated from a crack length increase from $0 \rightarrow 2 \ell$, the method gives a total energy value. Recalling that the ERR is defined as the energy that is released from growing an existing crack from $a$ to $a+d a$, the ARE values cannot be linked directly to interface strength values. A rather straightforward extension 
would be to perform an additional VCCT analysis [13, 17] which would then result in the desired ERR values. However, as explained by [13], the VCCT step in 3D requires symmetric orthonormal meshes around the crack front to get accurate results. For non-orthonormal meshes, corrections are proposed by Smith and Raju [19]. These corrections still require a mesh that should incorporate the shape of the crack front in an approximate way, i.e., the nodes should still be located on the (possibly curved) crack front. Clearly, for typical ARE calculations, i.e. energy calculations at each node within an interface, this is not feasible; (ii) although a length scale $\ell$ is present in the calculations (recall that an area is released instead of only one node), the mesh density should still be large to get converged results. This is caused by the fact that in three dimensions, a circular area should be released whereas a circular geometry cannot be captured accurately in regular grids. As a result, mesh insensitivity is in some cases only obtained with prohibitively large models.

Motivated by these issues, an enhanced ARE approach is proposed in this paper: calculate an accurate value for the ERR (which can be linked directly by measured interface strength values) using a two-step approach: (1) perform a global simulation in which the global degrees of freedom are calculated, without the presence of a crack; (2) perform a local simulation in which the (interpolated) global degrees of freedom are prescribed at the boundaries of the local model for any location $\mathrm{x}$ in a pre-defined interface $\mathcal{I}(\mathbf{x})$. This local model contains a proper threedimensional crack tip mesh thereby assuring reliable ERR values. This approach is depicted in Fig. 1 .

The model on the left corresponds ot the 'scale 1' model while the local crack tip mesh model is depicted on the right. Notice that in the crack tip model, the crack shape and size can be modified easily, while also different material interfaces and possibly other geometries (e.g., including a via) can be taken into account. Advantages of this two-step method are: (a) the calculated energy level is indeed the ERR value which can directly be linked to measured interface strength values; (b) the geometry of the local crack tip model is flexible: both the geometry of the crack front as well as the material geometry (in fact, the local crack tip mesh depicted in Fig. 1 is a very simple example). In the original ARE method, only penny-shaped

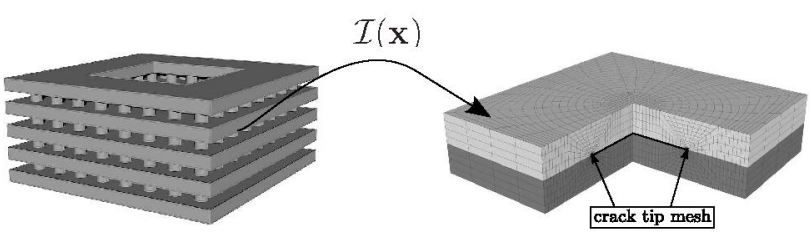

Figure 1: The proposed framework for the new ARE calculation: on the left, an example of the global model; on the right, an example of the local crack tip mesh model for any position $\mathrm{x}$ in a selected interface $\mathcal{I}(\mathrm{x})$ cracks could be used; (c) the main advantage of the original ARE method is preserved: flexible energy release calculation in any location at a pre-selected interface $\mathcal{I}(\mathbf{x})$ without modifying the original three-dimensional model.

In the next section, several analytical and numerical benchmarks will be discussed.

\section{Benchmarks}

In this section, several benchmark problems will be solved using the proposed enhanced ARE method as described in the previous section. The first three benchmarks are analytical benchmarks. In addition, two numerical benchmarks will be discussed. From a computational time point of view, the local model should be as small as possible, whereas from an accuracy point of view, these dimensions should be as large as possible. This trade-off will be studied in these benchmarks, in which the influence of the size of the local models on the resulting ERR values will be studied which provides some guidelines for the accuracy and application of the method.

\subsection{Penny-shaped crack in a uniform infinite medium}

As a first benchmark, a penny-shaped crack in a uniform infinite medium under remote tensile stress will be discussed. The geometry and boundary conditions are given in Fig.2(a). The analytical expression for the stress intensity factor is [20]

$$
K_{I}=\frac{2}{\pi} \sigma_{z z}^{\infty} \sqrt{\pi a}
$$

The following values have been assumed: $E=70000$ $\mathrm{MPa}, \nu=0.3, a=1 \mathrm{~mm}$ and $\sigma_{y y}^{\infty}=100 \mathrm{MPa}$. This results in $K_{I}=112.8 \mathrm{MPa} \sqrt{\mathrm{mm}}$ and $G=0.166 \mathrm{~N} / \mathrm{mm}$. The local crack model used in the local ARE simulations is depicted in Fig.2(b). Notice that the penny-shaped crack front has been modeled using an appropriate three-dimensional crack tip mesh.

To study the dependency of the size of the local crack model, the dimensions $x_{1} \times x_{2} \times x_{3}$ have been varied: $6 \times 6 \times 4,8 \times 8 \times 6$, and $10 \times 10 \times 8$. The results are given in Table 1. It can be concluded that these results are quite accurate, even though the global displacement solution vector, applied on the local model, does not correspond

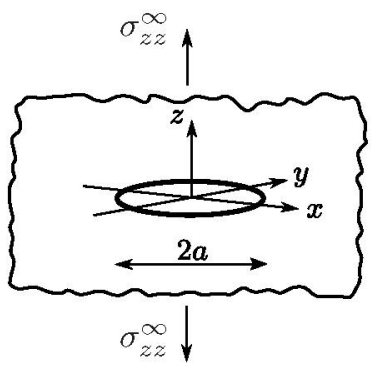

(a)

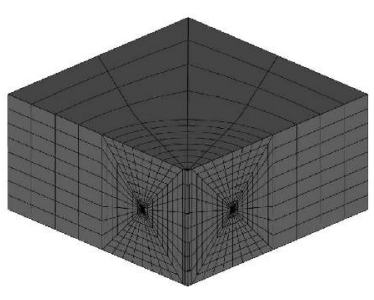

(b)
Figure 2: (a) Geometry and boundary conditions for the uniform penny-shaped crack benchmark, (b) the local crack model 
to a cracked geometry which confirms the potential of the proposed method. It is remarked that all results have been checked on mesh insensitivity.

Table 1: Results of the ERR of the uniform penny-shaped crack benchmark

\begin{tabular}{c|c|c} 
dimensions [mm & ERR value & error [\%] \\
\hline $6 \times 6 \times 4$ & 0.1454 & 12.4 \\
$8 \times 8 \times 6$ & 0.1592 & 4.0 \\
$10 \times 10 \times 8$ & 0.1640 & 1.0
\end{tabular}

The main application of the ARE method is the failure sensitivity of multi-layered complex three-dimensional structures in which the focus is on interface fracture. Therefore, the following benchmarks deal with the ERR calculation at bimaterial interfaces.

\subsection{Straight interface crack in an infinite plate}

The first benchmark of a bimaterial interface is the twodimensional one, introduced and solved by Rice [15], see Fig.3. The stress intensity factor is expressed by

$$
\mathbf{K}=\left(\sigma_{y y}^{\infty}+i \sigma_{x y}^{\infty}\right)(1+2 i \varepsilon) \sqrt{\pi a}(2 a)^{-i \varepsilon}
$$

The calculations have been performed with $E_{1}=70000$ $\mathrm{MPa}, \nu_{1}=0.2, E_{2}=35000 \mathrm{MPa}$ and $\nu_{2}=0.2$. For this material combination, $\beta=-0.125$ and $\varepsilon=0.04$. Again, $a=1 \mathrm{~mm}$ and $\sigma_{y y}^{\infty}=100 \mathrm{MPa}$. This results in $\mathbf{K}=177.57-9.26 i \mathrm{MPa} \sqrt{\mathrm{mm}}(\mathrm{mm})^{i \varepsilon}$ and $G=0.64$ $\mathrm{N} / \mathrm{mm}$. For this calculation, the local crack model has been generated and is illustrated in Fig.3(b). It should be noted that the analytical solution requires continuity of the normal strain component $\varepsilon_{x x}$ at the boundaries at infinity. This can be either taken into account by prescribing additional stresses at the edges, or to tie the nodal displacements at the edges in $x$-direction resulting in freely contracting, but straight, edges. The latter option has been used in the presented simulations. Also for this benchmark, the dimensions of the local model have been varied: $5 \times 5$, $10 \times 10$ and $14 \times 14$. The ERR is calculated from $(9)$ by using (5). The results are given in Table 2. The simulation results converge to the analytical solution which indicates that the method is quite accurate. This benchmark also illustrates that the method is not restricted to only one

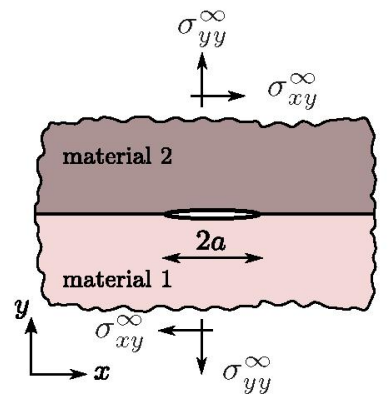

(a)

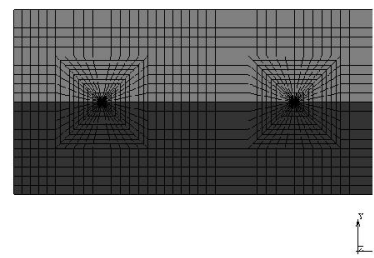

(b)
Figure 3: (a) Geometry and loading conditions of the Rice benchmark; (b) zoomed-in part of the local crack model
Table 2: Results of the ERR of the Rice benchmark

\begin{tabular}{c|c|c} 
dimensions & ERR value & error [\%] \\
\hline $5 \times 5$ & 0.567 & 11.4 \\
$10 \times 10$ & 0.605 & 5.4 \\
$14 \times 14$ & 0.622 & 2.8
\end{tabular}

kind of crack geometry, as was indeed the case in the previous ARE method where only penny-shaped cracks could be used.

\subsection{Penny-shaped crack in an infinite bimaterial}

The final analytical benchmark is a penny-shaped crack in a bimaterial interface. The geometry and boundary conditions are given in Fig.4. The analytical solution for the stress intensity factor $\mathbf{K}$ to this problem has been provided by Kassir and Bregman [12]:

$$
K_{1}+i K_{2}=2 \sigma_{z z}^{\infty} \frac{\Gamma(2+i \varepsilon)}{\Gamma\left(\frac{1}{2}+i \varepsilon\right)} \sqrt{a}(2 a)^{-i \varepsilon}
$$

where $\Gamma$ the Gamma function with complex argument.

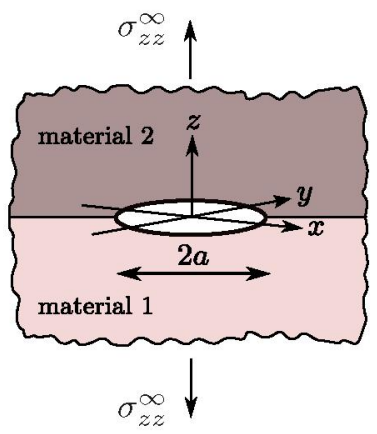

Figure 4: Geometry and boundary conditions for the bimaterial penny-shaped crack benchmark

The calculations have been performed with $E_{1}=70000$ $\mathrm{MPa}, \nu_{1}=0.2, E_{2}=35000 \mathrm{MPa}$ and $\nu_{2}=0.2$. Again, $a=1 \mathrm{~mm}$ and $\sigma_{y y}^{\infty}=100 \mathrm{MPa}$. This results in $\mathbf{K}=112.97-7.64 i \mathrm{MPa} \sqrt{\mathrm{mm}}(\mathrm{mm})^{i \varepsilon}$ and $G=0.260$ $\mathrm{N} / \mathrm{mm}$. For this calculation, the local crack model essentially equals the local model for the uniform penny-shaped crack, see Fig.2(b), except for the fact that now two materials are present in the model (see also the right picture in Fig.1). Also for this case, the analytical solution requires continuity of $\varepsilon_{r r}$ at the interface boundary at $r \rightarrow \infty$. This has been modeled with nodal tyings at the outer edges, such that (radial) contraction is allowed at the outer edges while the edges remain straight. Another way of achieving this, would be to prescribe additional stresses at the outer edges, as explained by Ayhan et al. [2]. The results of varying local model dimensions are given in Table 3. It can be seen that the ERR is calculated very accurately and converges to the analytical solution.

Indeed, the three benchmark cases confirm that the proposed two-level ARE method indeed captures the local stress field around any crack front thereby providing ac- 
Table 3: Results of the ERR of the bimaterial pennyshaped crack benchmark

\begin{tabular}{c|c|c} 
dimensions & ERR value & error [\%] \\
\hline $6 \times 6 \times 4$ & 0.233 & 10.2 \\
$8 \times 8 \times 6$ & 0.252 & 2.9 \\
$10 \times 10 \times 8$ & 0.258 & 0.6
\end{tabular}

curate ERR values. In the next section, a two- and a threedimensional application of the method will be illustrated.

\section{Application}

\subsection{Two-dimensional bond pad structure}

This application serves to illustrate the applicability in a more complex geometry: a two-dimensional cross-section of a characteristic part of a typical bond pad design, see Fig.5. The width of the model is $16 \mu \mathrm{m}$ and the height is $4.71 \mu \mathrm{m}$. A vertical displacement of $0.1 \mu \mathrm{m}$ is prescribed on the top whereas the displacements at the bottom and left side are suppressed. All materials are assumed linear elastic for this benchmark.

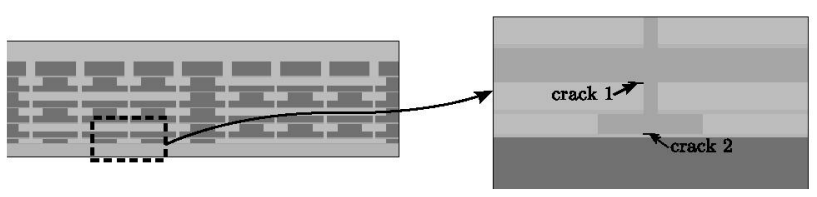

Figure 5: The two-dimensional bond pad model including a zoom of the two crack locations crack1 and crack 2

Clearly, for this case, no analytical solution exists. Therefore, two models have been made in which a crack with $a=0.13[\mu \mathrm{m}]$ (which corresponds to the diameter of a via) is inserted at two different locations, indicated in the right picture of Fig.5. Crack 1 is located at the metal/dielectricum interface, whereas crack 2 is inserted at the metal/hardmask interface. This approach is also used by Wang et al. [21] and Liu et al. [14] which clearly illustrates the disadvantage of the application of ERR calculations in the conventional way: a crack location (and geometry in 3D) has to be chosen and for each location, different models have to be generated. The thus calculated reference solution for crack 1 is $G=2.55 \mathrm{~N} / \mathrm{m}$ and for crack 2 is $G=5.76 \mathrm{~N} / \mathrm{m}$. These solutions are the converged values for several mesh density values. For the ARE approach, several local models have been generated with varying dimensions, indicated by Fig.6. Notice that here, only the location (e.g., an interface or as in this case, a single point) of the local model has to be provided in the global model. The

Table 4: Results of the errors in the ERR of the twodimensional bond pad structure benchmark

\begin{tabular}{c|c|c} 
dimensions & crack 1 [\%] & crack 2 [\%] \\
\hline small & 6.3 & 12.6 \\
medium & 3.9 & 4.5 \\
large & 1.1 & 1.5
\end{tabular}

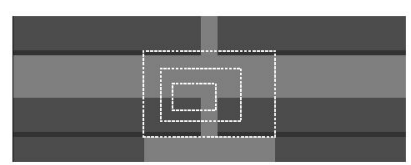

(a)

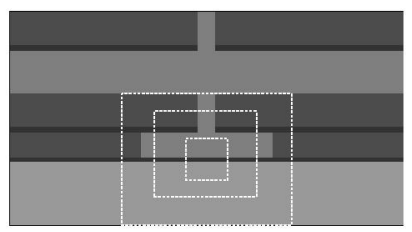

(b)
Figure 6: Different model sizes for the the twodimensional bond pad benchmark (a) for crack 1 and (b) for crack 2

crack tip meshes are equal to the mesh shown in Fig.3(b), only with different dimensions and materials. The results for both crack location are given in Table 4 . The regions illustrated in Fig.6 are denoted in the table by small, medium and large for convenience. From the results, it can be concluded that even for more complex geometries, the method is rather accurate, even for small local models.

\subsection{Three-dimensional bond pad structure}

An important application of the ARE method is the evaluation of the failure sensitivity of three-dimensional bond pad structures, which have already been evaluated with the existing ARE approach $[6,18]$, however, with the aforementioned shortcomings. As a benchmark example, the pad design as illustrated in Fig.7 will be used. Notice that the two-dimensional geometry from Section 5.1 is a crosssection of the three-dimensional geometry from Fig.7. For this benchmark, a penny-shaped crack of $2 a=0.13 \mu \mathrm{m}$ is inserted as depicted in the right picture of Fig.7. Analogous to the two-dimensional benchmark, a vertical displacement of $0.1 \mu \mathrm{m}$ is prescribed on top of the structure. Again, a reference solution is obtained by fully meshing the structure including an appropriate crack tip mesh. The converged ERR value is $G=0.707 \mathrm{~N} / \mathrm{m}$.

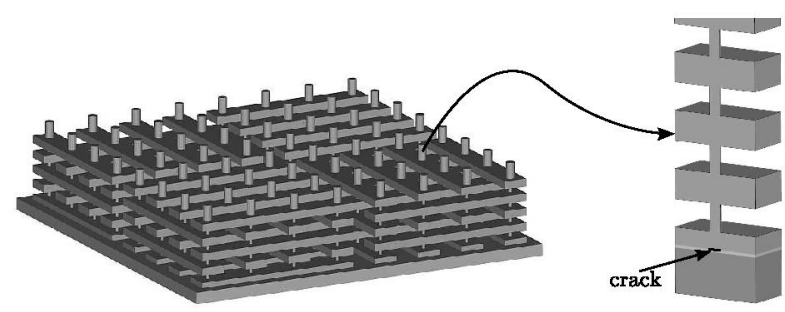

Figure 7: The three-dimensional bond pad model including a zoom of the crack location. For visualization purposes, the dielectrium and hardmask materials are removed from the pictures

For the ARE approach, three different local models have been generated with equal dimensions as the ones for the two-dimensional benchmark (crack 2) and indicated in Fig.6(b). The calculated ERR values are given in Table 5. It can be concluded that the results for the threedimensional benchmark are even more accurate than the two-dimensional benchmark. 
Table 5: Results of the ERR of the three-dimensional bond pad structure

\begin{tabular}{c|c|c} 
dimensions & ERR value & error [\%] \\
\hline small & 0.737 & 4.2 \\
medium & 0.720 & 1.8 \\
large & 0.711 & 0.6
\end{tabular}

\section{Conclusions}

In this paper, an enhanced version of the ARE method is proposed. In addition to the already efficient way of calculating energy values in an interface, already possible with the original ARE method, the calculated energy values are now accurate values for the ERR. Therefore, the values can directly be combined with interface strength values (or bulk strength values in the case for uniform materials). The method is based on a two-scale approach: at global level, the degrees of freedom are calculated without taking into account the cracked state whereas at local level, an arbitrary crack geometry is used for the calculation of the ERR. The benchmarks indicate that even for small local models, accurate ERR values are obtained. In this way, the geometry of the considered structures as well as the crack geometry and corresponding materials can be taken into account flexibly.

Future work will firstly focus on ARE computations within selected interfaces combined with already measured interface strength values by four-point bending tests and on the optimisation of the CPU time of the local models by means of numerical condensation. Secondly, the method can be combined with the dedicated cohesive zone model implementation by Van $\mathrm{Hal}$ et al. [7]: the ARE calculation will provide critical locations, whereas the cohesive zone models will subsequently be applied to model to transient crack propagation.

\section{References}

[1] Abdel Wahab M, de Roeck G (1995). "A 2-D five-noded finite element to model power singularity." International Journal of Fracture, 74:89-97.

[2] Ayhan A, Kaya A, Nied H (2006). "Analysis of threedimensional interface cracks using enriched finite elements." International Journal of Fracture, 142:255-276.

[3] Barsoum R (1976). "On the use of isoparametric finite elements in linear fracture mechanics." International Journal for Numerical Methods in Engineering, 10:23-37.

[4] van Driel W (2007). "Facing the challenge of designing for Cu/Low-k reliability." Microelectronics Reliability, accepted for publication.

[5] Ernst L, van Driel W, van der Sluis O, Corigliano A, Tay A, Iwamoto N, Yuen M (2007). "Fracture and delamination in microelectronics." Key Engineering Materials, accepted for publication.

[6] van Gils M, van der Sluis O, Zhang G, Janssen J, Voncken $\mathrm{R}$ (2007). "Analysis of $\mathrm{Cu} /$ low- $k$ bond pad delamination by using a novel failure index." Microelectronics Reliability, 47:179-186.

[7] van Hal B, Peerlings R, Geers M, van der Sluis O (2007). "Cohesive zone modeling for structural integrity analysis of IC interconnects." Microelectronics Reliability, accepted for publication.

[8] Hartfield C, Ogawa E, Park YJ, Chiu TC (2004). "Interface reliability assessments for Copper/low-k products." IEEE Transactions on Device and Materials Reliability, 4:129141.

[9] He M, Evans A, Hutchinson J (1994). "Crack deflection at an interface between dissimilar elastic materials: Role of residual stresses." International Journal of Solids and Structures, 31:3443-3455.

[10] Hutchinson J, Suo Z (1992). "Mixed mode cracking in layered materials." Advances in Applied Mechanics, 29:63191.

[11] Kanninen M, Popelar C (1985). Advanced Fracture Mechanics. Oxford University Press, New York.

[12] Kassir M, Bregman A (1972). "The stress-intensity factor for a penny-shaped crack between two dissimilar materials." Journal of Applied Mechanics, 39:308-310.

[13] Krueger R (2002). The virtual crack closure technique: history, approach and applications. NASA Report CR-2002211628.

[14] Liu X, Lane M, Shaw T, Simonyi E (2007). "Delamination in patterned films." International Journal of Solids and Structures, 44:1706-1718.

[15] Rice J (1988). "Elastic fracture mechanics concepts for interfacial cracks." Journal of Applied Mechanics, 55:98-103.

[16] Rice J, Sih G (1965). "Plane problems of cracks in dissimilar media." Journal of Applied Mechanics, 32:418- 423.

[17] Rybicki E, Kanninen M (1977). "A finite element calculation of stress intensity factors by a modified crack closure integral." Engineering Fracture Mechanics, 9:931-938.

[18] van der Sluis O, Engelen R, van Driel W, van Silfhout R, van Gils M (2007). "Efficient damage sensitivity analysis of advanced $\mathrm{Cu}$ Low- $k$ bond pad structures using Area Release Energy." Microelectronics Reliability, accepted for publication.

[19] Smith S, Raju I (1998). "Evaluation of stress-intensity factors using general finite-element models." In Fatigue and Fracture Mechanics: 29th Volume, ASTM STP 1321, edited by T Panontin, S Sheppard. American Society for Testing and Materials.

[20] Tada H, Paris P, Irwin G (1985). The stress analysis of cracks handbook. Paris Productions, St. Louis.

[21] Wang G, Ho P, Groothuis S (2005). "Chip-packaging interaction: a critical concern for $\mathrm{Cu} /$ low $k$ packaging." Microelectronics Reliability, 45:1079-1093.

[22] Zhang G, van Driel W, Fan X (2006). Mechanics of Microelectronics. Springer, Dordrecht, The Netherlands, ISBN $1-4020-4934-X$. 